\title{
openheart Bedside risk score for prediction of acute kidney injury after transcatheter aortic valve replacement
}

\author{
Nevena Zivkovic, ${ }^{1}$ Gabby Elbaz-Greener, ${ }^{1}$ Feng Qiu, ${ }^{2}$ \\ Yaron Arbel, ${ }^{3}$ Asim N Cheema, ${ }^{4}$ Danny Dvir, ${ }^{5}$ Paul Fefer, ${ }^{6}$ Ariel Finkelstein, ${ }^{3}$ \\ Stephen E Fremes, ${ }^{1}$ Sam Radhakrishnan, ${ }^{1}$ Josep Rodés-Cabau, ${ }^{7}$ Mony Shuvy, ${ }^{8}$ \\ Harindra C Wijeysundera ${ }^{1,2,9}$
}

To cite: Zivkovic $\mathrm{N}$, Elbaz-Greener G, Qiu F, et al. Bedside risk score for prediction of acute kidney injury after transcatheter aortic valve replacement. Open Heart 2018;5:e000777. doi:10.1136/ openhrt-2018-000777

Received 14 January 2018 Accepted 1 May 2018

\section{Check for updates}

${ }^{1}$ Schulich Heart Centre, Division of Cardiology, Sunnybrook Health Sciences Centre, University of Toronto, Toronto, Ontario, Canada

${ }^{2}$ Institute for Clinical Evaluative Sciences (ICES), Toronto, Ontario, Canada

${ }^{3}$ Department of Cardiology, Tel Aviv Medical Center, Sackler School of Medicine, Tel Aviv University, Tel Aviv, Israel ${ }^{4}$ Division of Cardiology, St. Michael's Hospital, Toronto, Ontario, Canada

${ }^{5}$ Division of Cardiology, St Paul's Hospital, Vancouver, British

Columbia, Canada

${ }^{6}$ Heart Institute, Sheba Medical Center, Tel HaShomer, Sackler Faculty of Medicine, Tel-Aviv University, Tel Aviv, Israel ${ }^{7}$ Quebec Heart and Lung Institute, Laval University, Quebec City, Quebec, Canada ${ }^{8}$ Heart Institute, Hadassah Hebrew University Medical Centre, Jerusalem, Israel ${ }^{9}$ Institute of Health Policy, Management and Evaluation, University of Toronto, Toronto, Ontario, Canada

Correspondence to Dr Harindra C Wijeysundera; Harindra.wijeysundera@ sunnybrook.ca

\section{ABSTRACT}

Background Acute kidney injury (AKI) is a common posttranscatheter aortic valve replacement (TAVR) complication associated with a poor prognosis. We sought to create a risk calculator using information that would be available during the work-up period.

Methods Data were obtained from a multicentre TAVR registry ( $n=1993)$ with cases from 1 January 2012 to 31 December 2015. We used logistic regression to create a risk calculator to predict AKI as defined by the Valve Academic Research Consortium Guidelines. We internally validated our risk calculator using bootstrapping, and evaluated model discrimination and calibration.

Results A simple risk score was derived with six variables, including New York Heart Association functional classification class 4, non-femoral access site, valve-invalve procedure, haemoglobin, creatinine clearance and weight in kilograms. The score was able to predict the absolute risk of AKI from $1 \%$ to $72 \%$. The model showed good discrimination with c-statistic 0.713 , with good agreement between predicted and observed AKI rates across quintiles of risk.

Conclusions This is the first risk calculator to assess post-TAVR risk of AKI. We found that information known pre-procedurally can be used to predict AKI. This may allow for more informed decision-making as well as identifying high-risk patients.

\section{INTRODUCTION}

Acute kidney injury (AKI) is a common complication following transcatheter aortic valve replacement (TAVR) that is associated with a very poor prognosis. Using the Valve Academic Research Consortium 2 (VARC-2) definition, the incidence of post-procedural AKI is $12 \%-45 \%,{ }^{1-12}$ with a meta-analysis estimation of $22.1 \pm 11.2 \%$ (13). AKI is associated with an increased risk of both shortterm and long-term mortality, independent of whether renal function returns to baseline levels, ${ }^{1-4911-17}$ and it increases the risk of other adverse events such as early myocardial infarction and dialysis. ${ }^{13}$

\section{Key questions}

What is already known about this subject?

- Acute kidney injury (AKI) is a common complication following transcatheter aortic valve replacement, occurring in an estimated $22.1 \pm 11.2 \%$ of patients. It is associated with a very poor prognosis.

What does this study add?

- We derived a simple to use risk score with six pre-procedurally known variables. The score was able to predict the absolute risk of AKI from $1 \%$ to $72 \%$.

How might this impact on clinical practice?

- A pre-procedure risk score identifies high-risk patients. It can help patients and clinicians in informed decision-making.

Previous studies evaluating the association between TAVR and AKI have identified various explanatory baseline, peri-procedural and procedural factors. However, there are no risk calculators in the literature to predict the absolute risk of AKI post-TAVR. A risk score to quantify the AKI risk that can be used during the work-up phase would aid clinicians and patients in the decision-making process. Indeed, such risk prediction tools are available for various cardiac procedures including cardiac surgery and percutaneous coronary interventions. ${ }^{18} 19$ These tools may be used in shared decision-making, in particular when TAVR is potentially futile due to multiple comorbidities. In such a setting, a risk of AKI that is sufficiently high to preclude a safe procedure would further reinforce the decision to avoid proceeding with TAVR. Alternatively, these tools would aid clinicians to identify patients who may benefit from prophylactic strategies to reduce the risk of AKI or who may require closer monitoring and care post-procedure. 
Accordingly, to address this gap in knowledge, the purpose of our study was to create a well-validated risk calculator using information that would be routinely available to clinicians during the TAVR work-up period. We hypothesise that such a model would accurately predict the risk of post-TAVR renal deterioration.

\section{METHODS}

We conducted a retrospective cohort study using patient data from six tertiary hospitals, three of which were located in Canada and three in Israel. Research ethics board approval was obtained from all centres. The need for individual patient consent was waived by all the institutional review boards.

Each site routinely maintains a TAVR registry prospectively, collecting detailed information on patients' baseline characteristics, procedure information and complications. We identified all patients receiving a TAVR procedure from 1 January 2012 to 31 December 2015. This time period was chosen in order to study a contemporary cohort, and after all sites had mature programmes and were beyond their learning curve phase. Patients were excluded if they were receiving dialysis at baseline, if they required emergency open heart surgery or if our main outcome of interest AKI could not be classified due to procedural death or missing data on creatinine. Patients who underwent emergency open heart surgery immediately post-TAVR were excluded because this complication would not be known during the diagnostic work-up phase.

The primary outcome of interest was stage 1, 2 or 3 AKI as defined by VARC-2 guidelines based on the difference between pre-procedural and highest post-procedural (within 7 days) creatinine values. ${ }^{20}$ Using the most liberal definition, any patient whose baseline creatinine increased by $\geq 0.3 \mathrm{mg} / \mathrm{dL}$ post-procedurally was classified as having AKI.

Potential covariates were chosen based on data availability and the previous AKI literature. Given our goal was to develop a tool that would be used in the work-up phase, we restricted covariates to only those that would be known pre-procedurally. This included patients' baseline characteristics, baseline comorbidities, blood laboratory measurements and echocardiographic readings. All baseline characteristics were collected as closely as possible prior to the procedure date. Creatinine clearance (CRCL) was calculated using the Cockroft-Gault equation. ${ }^{21}$ We used CRCL instead of estimated glomerular filtration rate (eGFR) because we did not have information on race, which is necessary for the calculation of eGFR. The only procedural characteristics were those that would be pre-planned, such as access site, and previous surgical aortic valve replacement resulting in a valve-in-valve procedure. Although we recorded contrast dye volume in millilitres, this was not used in the final calculator as it would not be available during the diagnostic phase of the TAVR work-up.
Chi-squared tests and t-tests were used to compare baseline characteristics of patients with and without AKI. For the list of potential covariates considered for model selection, please see table 1 . We excluded any variable with $>10 \%$ missing values and contrast dye volume. Using logistic regression, univariable associations were first determined for all potential covariates. Covariates with a $p$ value less than 0.1 on univariate analyses were considered potential candidates for the final multivariable logistic regression model. For variable selection, we used an automated approach, by generating 200 bootstrapped datasets, with patients chosen with replacement so that each bootstrapped dataset had the full sample size as our full cohort. Backward logistic regression models were performed on each individual dataset; covariates needed a $\mathrm{p}$ value of $<0.05$ to remain in the model. This resulted in 200 different multivariable models. We then determined the frequency by which each potential variables were in the 200 different multivariable models. Any covariate that was in $>50 \%$ of models was retained in the final model. Having selected the final covariates, we determined the parameter estimates in a complete dataset developed by multiple imputation. For any variables with $<10 \%$ missing data, we assumed that data were missing completely at random and created 200 imputed datasets using a fully conditional specification method. ${ }^{22}$ To convert the parameter estimates in the final model to score-based prediction rules, we used the methodology described by Sullivan and colleagues to obtain integer scores for each parameter. ${ }^{23}$

Internal validation of the model was performed using bootstrapping with unrestricted random sampling for 200 iterations. The c-statistic (ie, area under the receiver operating curve) was used to assess model discrimination while model calibration was evaluated with the Hosmer and Lemeshow $\chi^{2}$ statistic, as well as graphically by comparing predicted with observed risks by quintile. A $95 \%$ CI was calculated for both the c-statistic and Hosmer and Lemeshow $\chi^{2}$ statistic. All analyses were completed using SAS V.9.3 (SAS Institute, Cary, North Carolina, USA).

\section{RESULTS}

As seen in figure 1, a total of 2128 patients underwent TAVR at the six different hospitals between 1 January 2012 and 31 December 2015. We excluded 59 patients who were on dialysis pre-procedure and an additional 24 who had emergent open heart surgery. A total of 17 patients died during the procedure, and 35 had missing AKI data. The final cohort consisted of 1993 patients, of whom 318 (15.9\%) of patients had AKI, with 271 (13.6\%) having stage 1 AKI while $33(1.7 \%)$ and $14(0.7 \%)$ had stage 2 and 3 , respectively.

The patient and procedure characteristics of the cohort are shown in table 1 . The average age of the cohort was $82.0 \%$ and $51.1 \%$ were women. A minority of patients $(14.6 \%)$ had a non-femoral TAVR procedure and 106 $(5.3 \%)$ received a valve-in-valve procedure. About half (790 patients, $47.3 \%$ ) received conscious sedation. As 
Table 1 Patient and procedure characteristics of all patients and stratified by acute kidney injury

\begin{tabular}{|c|c|c|c|c|}
\hline Patient characteristics & All patients $(n=1993)$ & AKI $(n=318)$ & No AKI ( $n=1675)$ & $P$ values \\
\hline Age & $82.0 \pm 7.4$ & $82.4 \pm 6.9$ & $81.9 \pm 7.5$ & 0.24 \\
\hline Female & $1013(51.1 \%)$ & $157(49.5 \%)$ & $856(51.4 \%)$ & 0.55 \\
\hline Male & $971(48.9 \%)$ & $160(50.5 \%)$ & $811(48.7 \%)$ & \\
\hline NYHA class 4 & 346 (18.9\%) & 77 (25.8\%) & 269 (17.6\%) & 0.001 \\
\hline Atrial fibrillation & $641(32.2 \%)$ & $126(39.6 \%)$ & $515(30.8 \%)$ & 0.002 \\
\hline COPD & $351(17.6 \%)$ & $64(21.1 \%)$ & $287(17.2 \%)$ & 0.20 \\
\hline Diabetes & $712(35.7 \%)$ & $142(44.8 \%)$ & $570(34.0 \%)$ & $<0.001$ \\
\hline Hypertension & 1714 (86.0\%) & 275 (86.5\%) & 1439 (85.9\%) & 0.79 \\
\hline Lipids & $1442(72.6 \%)$ & $237(74.5 \%)$ & $1205(72.2 \%)$ & 0.40 \\
\hline PVD & $301(15.1 \%)$ & $51(16.0 \%)$ & $250(15.0 \%)$ & 0.63 \\
\hline Stroke or TIA & $323(16.2 \%)$ & $55(17.3 \%)$ & $268(16.0 \%)$ & 0.57 \\
\hline Prior open heart surgery & $404(24.4 \%)$ & $51(19.8 \%)$ & $353(25.2 \%)$ & 0.062 \\
\hline STS score & $5.8 \pm 5.0$ & $7.0 \pm 6.3$ & $5.6 \pm 5.4$ & $<0.001$ \\
\hline EuroSCORE & $7.2 \pm 7.3$ & $8.8 \pm 8.8$ & $6.8 \pm 6.9$ & $<0.001$ \\
\hline Pre-mean PG & $44.7 \pm 16.6$ & $43.0 \pm 15.7$ & $45.1 \pm 16.8$ & 0.043 \\
\hline LVEF $<40 \%$ & $217(11.2 \%)$ & $47(15.3 \%)$ & $170(10.5 \%)$ & 0.015 \\
\hline BMI & $27.2 \pm 5.6$ & $28.2 \pm 27.5$ & $27.1 \pm 26.8$ & 0.002 \\
\hline Weight (kg) & $73.0 \pm 16.2$ & $75.8 \pm 17.1$ & $72.5 \pm 15.9$ & $<0.001$ \\
\hline CRCL & $50.6 \pm 22.7$ & $44.6 \pm 21.4$ & $51.7 \pm 22.8$ & $<0.001$ \\
\hline Pre-TAVR creatinine $(\mu \mathrm{mol} / \mathrm{L})$ & $106.7 \pm 43.0$ & $127.1 \pm 53.7$ & $102.8 \pm 39.5$ & $<0.001$ \\
\hline Haemoglobin & $119.4 \pm 16.1$ & $114.2 \pm 15.5$ & $120.3 \pm 16.1$ & $<0.001$ \\
\hline Valve size & $26.5 \pm 2.4$ & $26.5 \pm 2.5$ & $26.4 \pm 2.4$ & 0.59 \\
\hline Conscious sedation & $790(47.3 \%)$ & $119(44.8 \%)$ & $497(47.8 \%)$ & 0.38 \\
\hline Non-femoral access site & $290(14.6 \%)$ & $76(23.9 \%)$ & $214(12.8 \%)$ & $<0.001$ \\
\hline Valve in valve procedure & $106(5.3 \%)$ & $8(2.5 \%)$ & $98(5.9 \%)$ & 0.015 \\
\hline Contrast dye (mL) & $125.3 \pm 63.5$ & $131.0 \pm 73.7$ & $124.2 \pm 61.3$ & 0.173 \\
\hline
\end{tabular}

AKI, acute kidney injury; BMI, body mass index; COPD, chronic obstructive pulmonary disease; CRCL, creatinine clearance; EuroSCORE, European System for Cardiac Operative Risk Evaluation; LVEF, left ventricle ejection fraction; NYHA, New York Heart Association functional classification; PG, peakgradient; PVD, peripheral vascular disease; STS score, Society of Thoracic Surgeons adult cardiac surgery risk score; TAVR, transcatheter aortic valve replacement; TIA, transient ischaemic attack.

seen in table 1 , there were important differences between the patients with AKI and those without.

Univariable associations between AKI and candidate covariates are shown in table 2. The final multivariable model is shown in table 3. Baseline kidney function, as measured by creatinine and CRCL, were both potential variables based on our a priori selection criteria. We elected to include only CRCL in our final model, as it had a stronger relationship, and it incorporates information about age and sex. The remaining variables in the final multivariable model were New York Heart Association functional classification (NYHA) class 4, non-femoral access site, valve-in-valve procedure, haemoglobin and weight measured in kilograms. Higher CRCL, higher haemoglobin and a valve-in-valve procedure all decreased the risk of AKI, while NYHA class 4, non-femoral access site and heavier weight were associated with higher risk.

The final risk scores for the model are shown in table 4, as are the absolute risks of post-TAVR AKI. Scores can in theory range from -3 to 35 points, which corresponds to a $1 \%$ to $72 \%$ risk of AKI. In our cohort of 1993 patients, the actual scores observed ranged from 0 to 33 points, with an associated risk of post-TAVR AKI ranging from $1 \%$ to $65 \%$.

The c-statistic showed a good discrimination with a mean value of 0.713 . The $\chi^{2}$ statistic showed a mean of 0.195 but a large range from 0 to 0.905 . When calibration was assessed graphically, there was excellent agreement between observed and predicted values (figure 2). The mean predicted risk for each quintile was $6.0 \%, 10.1 \%, 14.3 \%$, $19.4 \%$ and $31.1 \%$ compared with mean observed risks of $6.3 \%, 8.7 \%, 13.6 \%, 18.9 \%$ and $32.2 \%$, respectively.

\section{DISCUSSION}

In this study, we derived a simple six-factor risk score in order to predict post-TAVR AKI accurately. The score calculator incorporates information that is readily available in 
All TAVR patients from January 1, 2012 to December 31, $2015(n=2128)$

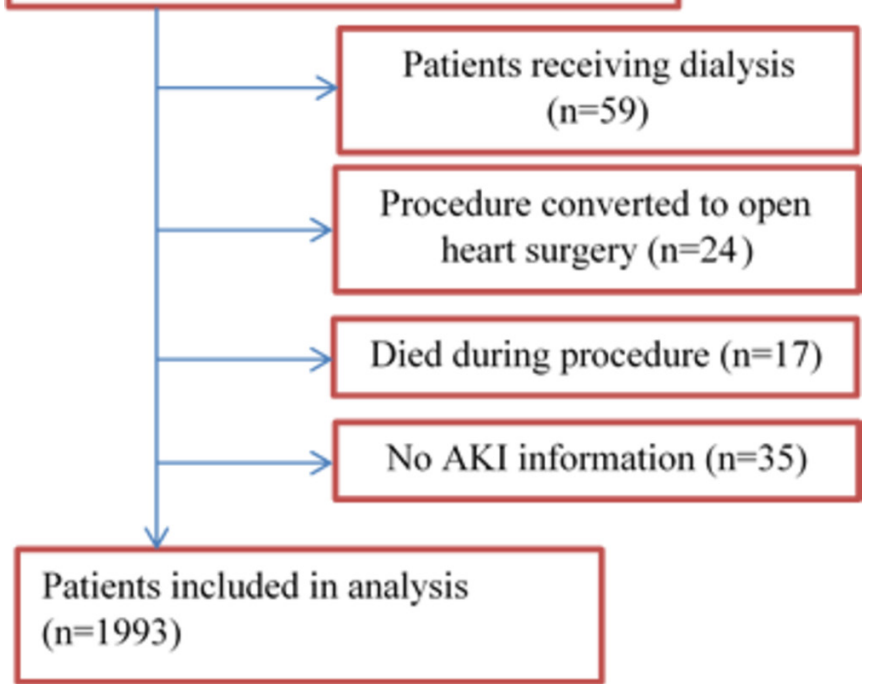

Figure 1 Patient selection flow chart. AKI, acute kidney injury; TAVR, transcatheter aortic valve replacement.

the diagnostic phase of the TAVR work-up, including procedure information about non-femoral access site and valve in valve as well pre-procedure haemoglobin, weight $(\mathrm{kg})$, CRCL and the presence of dyspnoea at rest (NYHA class 4). The predicted absolute risk for post-TAVR AKI based on the calculator can range from very low to extremely high risk ( $1 \%$ to $72 \%)$ and as such provides important information to clinicians and patients as they make decisions about proceeding with the procedure or to institute prophylactic strategies. ${ }^{24}$

Our findings were consistent with the previous literature on drivers of post-TAVR renal dysfunction. Low haemoglobin and worse baseline kidney function have been associated with AKI in multiple studies. ${ }^{4} 815$ 25-29 One study found higher BMI is a key predictor of kidney function decline ${ }^{28}$; although this was consistent in our univariable analysis, we found that weight was a stronger predictor in the multivariable model. We found that transapical access site increased risk of AKI, ${ }^{14}{ }^{26}$ as did any non-femoral access sites in our cohort. Based on this, we grouped all non-femoral access sites as a single category. Previous studies have also found an association with diabetes, EuroSCORE and poor left ventricular function. ${ }^{681730}$ To the best of our knowledge, ours is the only study to find that a valve-in-valve procedure, weight and NYHA class 4 are predictors of AKI post-TAVR. Notably, weight and NYHA class have previously been found to predict AKI or renal failure in cardiac surgery. ${ }^{19} 3132$ Prior cardiac surgery increased risk of acute renal failure among patients undergoing open heart surgery. ${ }^{28} 3334 \mathrm{In}$ contrast, we found valve-in-valve procedure was protective against AKI in TAVR.

The predictors we identified have face validity based on proposed pathophysiological mechanisms of AKI. A combination of haemodynamic, inflammatory,
Table 2 Univariable associations of baseline characteristics and acute kidney injury

\begin{tabular}{llc}
\hline Comorbidity & OR (95\% Cl) & P values \\
\hline Age & 1.01 (0.99 to 1.03$)$ & 0.24 \\
\hline Male & $1.08(0.85$ to 1.37$)$ & 0.55 \\
\hline NYHA class 4 & $1.27(1.1$ to 1.47$)$ & 0.001 \\
\hline Atrial fibrillation & $1.48(1.15$ to 1.89$)$ & 0.002 \\
\hline COPD & $1.22(0.89$ to 1.64$)$ & 0.20 \\
\hline Diabetes & $1.57(1.23$ to 2.01$)$ & $<0.001$ \\
\hline Hypertension & $1.05(0.75$ to 1.50$)$ & 0.79 \\
\hline Lipids & $1.12(0.86$ to 1.48$)$ & 0.40 \\
\hline PVD & $1.09(0.78$ to 1.5$)$ & 0.63 \\
\hline Stroke or TIA & $1.1(0.79$ to 1.5$)$ & 0.57 \\
\hline Prior open heart surgery & $0.73(0.53$ to 1.02$)$ & 0.063 \\
\hline STS score & $1.04(1.02$ to 1.07$)$ & $<0.001$ \\
\hline EuroSCORE & $1.03(1.02$ to 1.05$)$ & $<0.001$ \\
\hline Pre-mean PG & $0.99(0.98$ to 1.00$)$ & 0.048 \\
\hline LVEF <40\% & $1.54(1.09$ to 2.18$)$ & 0.016 \\
\hline BMI & $1.03(1.01$ to 1.05$)$ & 0.002 \\
\hline Weight (kg) & $1.01(1.01$ to 1.02$)$ & 0.001 \\
\hline CRCL & $0.98(0.98$ to 0.99$)$ & $<0.001$ \\
\hline Pre-TAVR creatinine & $1.01(1.01$ to 1.01$)$ & $<0.001$ \\
\hline Haemoglobin & $0.98(0.97$ to 0.98$)$ & $<0.001$ \\
\hline Valve size & $1.01(0.97$ to 1.07$)$ & 0.58 \\
\hline Conscious sedation & $0.83(0.64$ to 1.08$)$ & 0.17 \\
\hline Transapical access site & $2.00(1.40$ to 2.81$)$ & $<0.001$ \\
\hline Non-femoral access site & $2.14(1.59$ to 2.87$)$ & $<0.001$ \\
\hline Valve in valve & $0.42(0.18$ to 0.81$)$ & 0.019 \\
\hline BMI $)$ & \\
\hline
\end{tabular}

BMI, body mass index; COPD, chronic obstructive pulmonary disease; CRCL, creatinine clearance; EuroSCORE, European System for Cardiac Operative Risk Evaluation; LVEF, left ventricle ejection fraction; NYHA, New York Heart Association functional classification; PG, peak gradient; PVD, peripheral vascular disease; STS score, Society of Thoracic Surgeons adult cardiac surgery risk score; TAVR, transcatheter aortic valve replacement; TIA, transient ischaemic attack.

nephrotoxic and embolic factors may impair renal function and lead to a systemic injury. ${ }^{35}$ Having worse kidney function at baseline, as captured by a lower CRCL,

Table 3 Final multivariable model for predictors of acute kidney injury

\begin{tabular}{llc} 
Model parameter & OR $(95 \%$ Cl) & P values \\
\hline NYHA class 4 & $1.18(1.01$ to 1.40$)$ & 0.041 \\
Non-femoral access site & $1.52(1.30$ to 1.77$)$ & $<0.001$ \\
Valve-in-valve procedure & $0.58(0.40$ to 0.84$)$ & 0.004 \\
Haemoglobin & $0.98(0.97$ to 0.99$)$ & $<0.001$ \\
CRCL & $0.98(0.97$ to 0.98$)$ & $<0.001$ \\
Weight $(\mathrm{kg})$ & $1.03(1.02$ to 1.04$)$ & $<0.001$ \\
\hline
\end{tabular}

CRCL, creatinine clearance; NYHA, New York Heart Association functional classification. 
Aortic and vascular disease

\begin{tabular}{|c|c|c|}
\hline Risk factor & Categories & Points \\
\hline \multirow[t]{2}{*}{ NYHA class } & Class $1-3$ & 0 \\
\hline & Class 4 & 1 \\
\hline \multirow[t]{2}{*}{ Non-femoral access site } & No & 0 \\
\hline & Yes & 3 \\
\hline \multirow[t]{2}{*}{ Valve-in-valve procedure } & No & 0 \\
\hline & Yes & -3 \\
\hline \multirow[t]{6}{*}{ Haemoglobin } & $<100$ & 7 \\
\hline & $100-109$ & 6 \\
\hline & $110-119$ & 4 \\
\hline & $120-129$ & 3 \\
\hline & $130-139$ & 2 \\
\hline & $140+$ & 0 \\
\hline \multirow[t]{8}{*}{ Creatinine clearance } & $<25$ & 13 \\
\hline & $25-34$ & 12 \\
\hline & $35-44$ & 11 \\
\hline & $45-54$ & 9 \\
\hline & $55-64$ & 7 \\
\hline & $65-74$ & 6 \\
\hline & $75-84$ & 4 \\
\hline & $85+$ & 0 \\
\hline \multirow[t]{9}{*}{ Weight (kg) } & $<55$ & 0 \\
\hline & $55-59$ & 2 \\
\hline & $60-64$ & 3 \\
\hline & $65-69$ & 4 \\
\hline & $70-74$ & 5 \\
\hline & $75-80$ & 6 \\
\hline & $80-84$ & 7 \\
\hline & $85-89$ & 8 \\
\hline & $90+$ & 11 \\
\hline
\end{tabular}

\begin{tabular}{ccll}
\hline Points & $\begin{array}{l}\text { Estimate } \\
\text { of risk }\end{array}$ & Points & $\begin{array}{l}\text { Estimate } \\
\text { of risk }\end{array}$ \\
\hline-3 & $1 \%$ & 17 & $12 \%$ \\
-2 & $1 \%$ & 18 & $14 \%$ \\
\hline-1 & $1 \%$ & 19 & $16 \%$ \\
\hline 0 & $1 \%$ & 20 & $18 \%$ \\
\hline 1 & $1 \%$ & 21 & $21 \%$ \\
\hline 2 & $1 \%$ & 22 & $24 \%$ \\
\hline 3 & $1 \%$ & 23 & $27 \%$ \\
\hline 4 & $2 \%$ & 24 & $30 \%$ \\
\hline 5 & $2 \%$ & 25 & $34 \%$ \\
\hline 6 & $2 \%$ & 26 & $37 \%$ \\
\hline 7 & $3 \%$ & 27 & $41 \%$ \\
\hline 8 & $3 \%$ & 28 & $45 \%$ \\
\hline 9 & $4 \%$ & 29 & $49 \%$ \\
10 & $4 \%$ & 30 & $53 \%$ \\
\hline & & & \\
\hline
\end{tabular}

\begin{tabular}{lcll|}
\hline Table 4 & Continued & & \\
\hline Points & $\begin{array}{c}\text { Estimate } \\
\text { of risk }\end{array}$ & Points & $\begin{array}{l}\text { Estimate } \\
\text { of risk }\end{array}$ \\
\hline 11 & $5 \%$ & 31 & $58 \%$ \\
12 & $6 \%$ & 32 & $61 \%$ \\
13 & $7 \%$ & 33 & $65 \%$ \\
14 & $8 \%$ & 34 & $69 \%$ \\
15 & $9 \%$ & 35 & $72 \%$ \\
16 & $10 \%$ & & \\
\hline
\end{tabular}

NYHA, New York Heart Association functional classification.

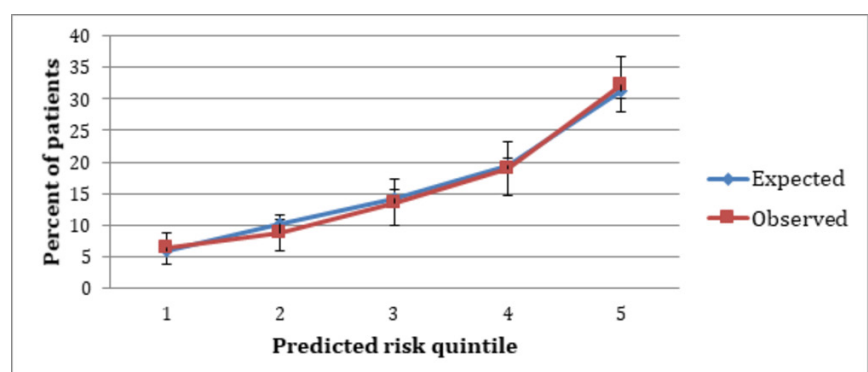

Figure 2 Expected versus observed percentage of patients with acute kidney injury stratified by predicted risk quintile.

increases kidney susceptibility to toxic peri-procedural events. Hypoperfusion to kidneys causing ischaemia may lead to AKI, explaining why a patient with poorer baseline cardiac function (NYHA class 4) or lower values of haemoglobin may be at higher risk.

Damage to kidneys via atherosclerotic plaque has also been previously described, and peripheral vascular disease has been a well-known cause of AKI in open heart surgery. ${ }^{32}$ Although we did not find that patients with peripheral vascular disease were at increased risk of TAVR, we did identify non-transfemoral TAVR access as a strong predictor of AKI. It is likely that the underlying mechanism for this observation was that the non-femoral access patients had severe peripheral vascular disease precluding a transfemoral TAVR, putting them at higher risk for AKI.

Weight has a complex relationship with AKI, in that it was an important predictor, despite being part of the CRCL calculation, suggesting that its relationship is non-linear. Although our work does not suggest an underlying pathophysiological mechanism for the relationship between AKI with weight, as noted, this has been observed with cardiac surgery also. ${ }^{32}$ Finally, valve-in-valve procedure reduces the risk of AKI. We hypothesise that this may be because valvein-valve procedures are generally shorter and likely require less contrast media.

Our risk score was designed to be independent of factors that would only be known after the TAVR, such as contrast volume. This design allows for broader use, specifically during the decision-making time period for both clinicians and patients. We elected to group AKI stage $1-3$, given the relatively small number of events. However, it is likely that information specific to the most 
severe forms of AKI, including the need for dialysis, would have the greatest impact on decision-making. Less than $1 \%$ of patients with AKI were in this most severe category, and thus our study was underpowered to evaluate this particular population. Nonetheless, further research to develop risk scores for severe post-TAVR AKI is warranted.

Our study must be interpreted in the context of several limitations that merit discussion. This is a retrospective study, so we cannot imply causality, nor can we be certain that all confounding factors were accounted for. Concomitant mitral regurgitation and myocardial infarction have previously been identified as independent predictors, but we did not have the data to explore this. ${ }^{30}$ In addition, we have a very small sample size of non-femoral or non-transapical access sites, so there should be caution in interpreting the effects of alternative access sites. We did not include certain factors such as contrast volume, which likely impact AKI incidence, as that would not be known at the time of decision-making. Nonetheless, minimising contrast in patients with a higher risk of AKI would undoubtedly impact postTAVR AKI. Finally, although we used the VARC-2 definition of AKI, ${ }^{20}$ we were unable to measure urine output. A reduced urine output, with no increase in creatinine, is sufficient to classify AKI in the VARC-2 criteria. VARC-2 also allows for the change in creatinine to take place of the course of 7 days post-TAVR. Although we recorded all creatinine data that were available first week while patients were still in hospital, it is possible that some patients developed AKI in this time frame after they were discharged. Because of these diagnostic considerations, we may have misclassified some patients who had AKI.

Our study shows that it is possible to create an AKI calculator using only pre-procedurally known variables. We believe that this risk calculator will be of interest to the general TAVR community for use in the diagnostic work-up phase, by allowing a better understanding of the risks and rewards offered by TAVR during the decision-making process.

Funding The authors have not declared a specific grant for this research from any funding agency in the public, commercial or not-for-profit sectors.

Competing interests None declared.

Patient consent Not required.

Ethics approval Sunnybrook Hospital, St Michael's Hospital, Quebec Heart and Lung Institute, Tel Aviv Medical Centre, Hadassah Hebrew University Medical Centre and Sheba Medical Center.

Provenance and peer review Not commissioned; externally peer reviewed.

Data sharing statement № additional data are available.

Open Access This is an Open Access article distributed in accordance with the Creative Commons Attribution Non Commercial (CC BY-NC 4.0) license, which permits others to distribute, remix, adapt, build upon this work non-commercially, and license their derivative works on different terms, provided the original work is properly cited and the use is non-commercial. See: http://creativecommons.org/ licenses/by-nc/4.0/

(c) Article author(s) (or their employer(s) unless otherwise stated in the text of the article) 2018. All rights reserved. No commercial use is permitted unless otherwise expressly granted.

\section{REFERENCES}

1. Barbash IM, Ben-Dor I, Dvir D, et al. Incidence and predictors of acute kidney injury after transcatheter aortic valve replacement. Am Heart J 2012;163:1031-6.

2. Carrabba N, Valenti R, Migliorini A, et al. Prognostic value of myocardial injury following transcatheter aortic valve implantation. Am J Cardiol 2013;111:1475-81.

3. Nuis RJ, Van Mieghem NM, Tzikas A, et al. Frequency, determinants, and prognostic effects of acute kidney injury and red blood cell transfusion in patients undergoing transcatheter aortic valve implantation. Catheter Cardiovasc Interv 2011;77:881-9.

4. Sinning JM, Ghanem A, Steinhäuser $\mathrm{H}$, et al. Renal function as predictor of mortality in patients after percutaneous transcatheter aortic valve implantation. JACC Cardiovasc Interv 2010;3:1141-9.

5. Konigstein M, Ben-Assa E, Banai S, et al. Periprocedural bleeding, acute kidney injury, and long-term mortality after transcatheter aortic valve implantation. Can J Cardiol 2015;31:56-62.

6. Nuis RJ, Rodés-Cabau J, Sinning JM, et al. Blood transfusion and the risk of acute kidney injury after transcatheter aortic valve implantation. Circ Cardiovasc Interv 2012;5:680-8.

7. Yamamoto M, Hayashida K, Mouillet G, et al. Renal function-based contrast dosing predicts acute kidney injury following transcatheter aortic valve implantation. JACC Cardiovasc Interv 2013;6:479-86.

8. Alassar A, Roy D, Abdulkareem N, et al. Acute kidney injury after transcatheter aortic valve implantation: incidence, risk factors, and prognostic effects. Innovations 2012;7:389-93.

9. Scherner M, Madershahian N, Ney S, et al. Focus on the surgical approach to transcatheter aortic valve implantation: complications, outcome, and preoperative risk adjustment. J Thorac Cardiovasc Surg 2015;150:841-9.

10. Saia F, Ciuca C, Taglieri N, et al. Acute kidney injury following transcatheter aortic valve implantation: incidence, predictors and clinical outcome. Int J Cardiol 2013;168:1034-40.

11. Covolo E, Saia F, Napodano M, et al. Comparison of balloonexpandable versus self-expandable valves for transcatheter aortic valve implantation in patients with low-gradient severe aortic stenosis and preserved left ventricular ejection fraction. Am J Cardiol 2015;115:810-5.

12. Codner P, Orvin K, Assali A, et al. Long-term outcomes for patients with severe symptomatic aortic stenosis treated with transcatheter aortic valve implantation. Am J Cardiol 2015;116:1391-8.

13. Gargiulo G, Sannino A, Capodanno D, et al. Impact of postoperative acute kidney injury on clinical outcomes after transcatheter aortic valve implantation: a meta-analysis of 5,971 patients. Catheter Cardiovasc Interv 2015;86:518-27.

14. Bagur R, Webb JG, Nietlispach F, et al. Acute kidney injury following transcatheter aortic valve implantation: predictive factors, prognostic value, and comparison with surgical aortic valve replacement. Eur Heart J 2010;31:865-74.

15. Elhmidi Y, Bleiziffer S, Piazza N, et al. Incidence and predictors of acute kidney injury in patients undergoing transcatheter aortic valve implantation. Am Heart J 2011;161:735-9.

16. Voigtländer L, Schewel J, Martin J, et al. Impact of kidney function on mortality after transcatheter valve implantation in patients with severe aortic valvular stenosis. Int J Cardiol 2015;178:275-81.

17. Liao YB, Deng XX, Meng Y, et al. Predictors and outcome of acute kidney injury after transcatheter aortic valve implantation: a systematic review and meta-analysis. Eurolntervention 2017;12:2067-74.

18. Tsai TT, Patel UD, Chang TI, et al. Validated contemporary risk model of acute kidney injury in patients undergoing percutaneous coronary interventions: insights from the National Cardiovascular Data Registry Cath-PCI Registry. J Am Heart Assoc 2014;3:e001380.

19. Ng SY, Sanagou M, Wolfe R, et al. Prediction of acute kidney injury within 30 days of cardiac surgery. J Thorac Cardiovasc Surg 2014;147:1875-83.

20. Kappetein AP, Head SJ, Généreux P, et al. Updated standardized endpoint definitions for transcatheter aortic valve implantation: the Valve Academic Research Consortium-2 consensus document (VARC-2). Eur J Cardiothorac Surg 2012;42:S45-60.

21. Cockcroft DW, Gault MH. Prediction of creatinine clearance from serum creatinine. Nephron 1976;16:31-41.

22. Van Buuren S, Brand JPL, Groothuis-Oudshoorn CGM, et al. Fully conditional specification in multivariate imputation. J Stat Comput Simul 2006;76:1049-64.

23. Sullivan LM, Massaro JM, D'Agostino RB. Presentation of multivariate data for clinical use: The Framingham Study risk score functions. Stat Med 2004;23:1631-60.

24. Arbel Y, Ben-Assa E, Halkin A, et al. Forced diuresis with matched hydration in reducing acute kidney injury during transcatheter aortic 
valve implantation (Reduce-AKI): study protocol for a randomized sham-controlled trial. Trials 2014;15:262.

25. Arai T, Morice MC, O'Connor SA, et al. Impact of pre- and postprocedural anemia on the incidence of acute kidney injury and 1 -year mortality in patients undergoing transcatheter aortic valve implantation (from the French Aortic National CoreValve and Edwards 2 [FRANCE 2] Registry). Catheter Cardiovasc Interv 2015;85:1231-9.

26. Aregger F, Wenaweser P, Hellige GJ, et al. Risk of acute kidney injury in patients with severe aortic valve stenosis undergoing transcatheter valve replacement. Nephrol Dial Transplant 2009;24:2175-9.

27. Gargiulo G, Capodanno D, Sannino A, et al. Moderate and severe preoperative chronic kidney disease worsen clinical outcomes after transcatheter aortic valve implantation: meta-analysis of 4992 patients. Circ Cardiovasc Interv 2015;8:e002220.

28. Mehta RH, Grab JD, O'Brien SM, et al. Bedside tool for predicting the risk of postoperative dialysis in patients undergoing cardiac surgery. Circulation 2006;114:2208-16.

29. Barbanti M, Latib A, Sgroi C, et al. Acute kidney injury after transcatheter aortic valve implantation with self-expanding CoreValve prosthesis: results from a large multicentre Italian research project. Eurolntervention 2014;10:133-40.
30. Van Linden A, Kempfert J, Rastan AJ, et al. Risk of acute kidney injury after minimally invasive transapical aortic valve implantation in 270 patients. Eur J Cardiothorac Surg 2011;39:835-43. discussion 42-3.

31. Duthie FA, McGeehan P, Hill S, et al. The utility of the additive EuroSCORE, RIFLE and AKIN staging scores in the prediction and diagnosis of acute kidney injury after cardiac surgery. Nephron Clin Pract 2014;128:29-38.

32. Birnie K, Verheyden V, Pagano D, et al. Predictive models for kidney disease: improving global outcomes (KDIGO) defined acute kidney injury in UK cardiac surgery. Crit Care 2014;18:606.

33. Thakar CV, Arrigain S, Worley S, et al. A clinical score to predict acute renal failure after cardiac surgery. J Am Soc Nephrol 2005;16:162-8.

34. Wijeysundera DN, Karkouti K, Dupuis JY, et al. Derivation and validation of a simplified predictive index for renal replacement therapy after cardiac surgery. JAMA 2007;297:1801-9.

35. Siontis GC, Praz F, Pilgrim T, et al. Transcatheter aortic valve implantation vs. surgical aortic valve replacement for treatment of severe aortic stenosis: a meta-analysis of randomized trials. Eur Heart J 2016;37:3503-12. 